ence leads me to regard quinine and nux vomica as possessing similar powers. If, as I believe, we may regard the physiological action of any drug as determining its, therapeutical value and property, what we want are exact physiological experiments tested by clinical observations. There can, I suppose, be no doubt that ergot acts directly upon the involuntary unstriped muscular fibre wherever it is met with that its action, therefore, is by no means limited to the uterus, and that it has a very distinct effect upon the muscular coats of arteries and capillaries ; hence, in poisonous doses, it causes such contraction of these vessels that the blood is driven into the veins, which accordingly become distended, and the patient assumes quite a livid hue. This phenomenon I have observed again and again, and the explanation must, I think, be that which I have just given. Here, then, we have a fact which might be turned to therapeutical account.

As an example of a pelvic anodyne, with special reference to the ovaries, I know of none that can compare with conium, or, better still, with the alkaloid conia, used in the form of vaginal pessary. In all cases, whetter neuralgic or inflammatory, in which the ovaries are the seat of pain, conia is, to my mind, quite a specific. No drug that I know of acts with equal certainty and success. Lastly, I suppose we are all agreed that bromide of potassium exercises most powerful influence upon the ovaries. No drug, in my experience, can equal it in controlling ovarian mucorrhagia ; it not only limits the flow in these particular cases, but it seems, also, to exercise a distinct and controlling influence upon ovulation itself; hence, if $\mathrm{Mr}$. Lawson Tait will allow me to say so, its value in checking ovarian mucorrhagia, so far as regards its too frequent periodicity, for it certainly increases the length of the menstrual interval ; in other words, it controls too frequent menstruation, or, as I should say, too frequent ovulation. Now, we want more of such remedies as those I have mentioned, remedies which we can prescribe with almost absolute knowledge of their therapeutical value and effect, and we may look to the experience of the Fellows of this Society to supply many more examples of this kind. It will be by papers on such subjects, giving us exact experiments and observations, more probably than by the record of our surgical experiences, that this Society will fulfil what I hope may be regarded as one, if not the chief, of its functions, namely, to educate the mass of our professional brethren in the therapeutical branch of their calling in these particular cases. Nothing, I think, could more fitly demonstrate its utility than the record of such experience, and such work as this would, I am sure, earn and receive the gratitude both of the profession and the public.

Gentlemen, I trust you will forgive me for having detained you so long. I have encleavoured as briefly as I could, and I know very imperfectly, to give a slight sketch of the work and office of this Society, as the subject presents itself to my mind. No one, I think, will venture to deny that, with such objects in view, the founding of this Society, if it be true to its mission, is a great and useful work. I earnestly hope that in all our discussions we shall ever keep in view this primary purpose, and that we shall meet here with the everpresent consciousness that our work is not only scientific, but noble and grand, as all truly scientific work is, and must be, from the very nature of the case. I trust that the brilliancy of our discussions will never be tarnished by personalities, and that we shall ever bear in mind that criticism in matters of opinion, be it ever so sharp and incisive, is not a matter which need or should give any personal offence. $\mathrm{He}$ who feels offended at such criticism is thinking more of himself than his work; be it ours to think more of our work than ourselves, so that when we shall have passed away our work may still live on, and do good suit and service in the cause of suffering humanity, for then, assuredly, we shall not have lived and worked in vain.

The Unfounded Accusation against a Medical Man.-In our issue of the 7 th instant, we drew attention, under the above heading to the case of Parker $v$. Whitefoord, tried in the Court of Queen's Bench, before Mr. Justice A. I. Smith and a common jury. To the opinion then expressed we refer our readers. We now learn that, so far from Mr. Whitefoord beingr exonerated from pecuniary liabilitics, he has had to pay, not only the award of $£ 10$ damages, but his own and the costs of the plaintiff's solicitors, which have been reduced by the taxing master from $£ 10215 \mathrm{~s}$. $4 \mathrm{~d}$. to $£ 7117 \mathrm{~s}$. $10 \mathrm{~d}$. It appears to us that no other course was open to this gentleman but to defend his reputation from the aspersion on his character which the plaintifl and her adviser sought to cast on him; and, from the correspondence put before us as to the plaintiff's mental condition, and her immediate surroundings, we feel that Mr. Whitefoord merits, at the hands of the profession, their sincere sympathy, especially after the expression of opinion of the jury, concurred in, as it was, by the jutge.
REMARKS

or

\section{THE ETIOLOGY OF ASIATIC CHOLERA:}

A Paper read before the Royal Medical and Chirurgical Socicty, in reference to some points stated in the President's Address.

BY E. KLEIN, M.D., F.R.S.,

Lecturer on Histology at St. Bartholomew's Hospital.

Mr. President and Gentlemen, - In this most interesting address, to which we have just now been listening, there are two passages, referring to the etiology of cholera, about which I will take the liberty of making a few remarks. 1. The first is: "Cholera is believed to result from the action of a specific contagium or morbid infecting agen $2 y$, which may enter the system, either with the air through the lungs, or with food, water, or other liquids through the alimentary canal ;" and 2, "The intestinal discharges are the means by which the disease is conveyed from the sick to the healthy. In this respect, there is a close resemblance between cholera and enteric fever.'

1. Cholera, when it appears and spreads in Europe, is the result of importation from an infected locality. For this there is such overwhelming evidence, that it is not necessary to enter into the details of the history of the epidemics that visited the Eurnpean countries. Now, what is it that is imported, or, with o!lu? irords, what is the infective agency? That cholera has been improted by linen and clothes coming from an infected locality, of this there are several well established examples; but, amongst these, there are some, like the one mentioned by Professor Hirsch (Conferenz zur Erörterung der Cholerafrage, Berlin. Klin. Woch., No. 31, 1884)-when articles (linen, clothes, sweets) brought from an infected country (St. Louis, U.S.A.) have become the source of infection in Mïhlhausen (Thuringia), in 1873-in which the articles had not been soiled by cholera-patients. And, similarly, there are cases known where infection was carried from one locality into another by persons themselves not sick with cholera. From this it follows that the infective agency is not necessarily associated with choleraic dejecta, but that, independently of these, it may pass from an infected locality into a new one.

The infective agent must be a living entity, since it is possessed of the power of self-multiplication. After the infective agent is introduced into a locality, cholera-cases may occur within short intervals of time in distant centres, and from here spread to wider areas; hence the conclusion is inevitable, that that agent has, since its introduction, undergone self-multiplication, has spread into those distint centres, and here has produced infection. Since no chemical substance is capable of fulfilling this condition of self-multiplication, it lollows that the infective agent must be a living entity.

Two alternatives present themselves:- $-a$, the infective agent and actual virus are identical ; and $b$, the infective agent is not the vin us itself, but is the producer of the virus. In the first instance, we liave to deal with an organism which, having entered a susceptible person, here multiplies to an enormous extent and produces the disease; the morbid products, themselves charged with the new brood, are themselves: the contagium. Such is the case in the true infectious diseases, like anthrax, tuberculosis, gonorrhea, erysipelas, glanders, etc. In the second instance, the micro-organisms are extraneous, but produce a ferment which is the virus; the micro-organism does not enter the body, but lives and multiplies outside this, and as a result of its activity procinces a chemical ferment, which when introduced into the system acts as the virus. This is exemplified in cases of septic poisons, the ptomaines. The disease set up by these ferments is then equivalent to a chemical poisoning. In these instances, as a necessary conceifucuce, the morbid products contain neither the orranism nor the virus.

Now, does what is known of cholera fulfil the conditions of the former or latter group of diseases?

I maintain that cholera cannot helong to the first croun of diseases for these reasons. The general opinion as to the incubation-period of cholera is, that it varies between one and two days; but there arc several carefully observed cases, where the incubation could not have extended to more than from half an hour to a few hours (see Professor Drasche's account of cholera-cases in a laundry in Vienna, 1873, as described in a pamphlet, Der Pilzfund bei der Cholera, Vienna, 1884). 
It is quite out of question that, if the cholera-virus itself were a living entity, that is, a micro-organism, this having entered the system of a person could, within so short a space of time, have multiplied to such an extent as to set up the disease. These cases of rapid infection can only be explained by assuming that the virus is a ferment which is formed outside the body, which is absorbed by the system, and produces acute prisoning. The whole course of the disease and the symptoms eminently favour the theory that it is due to a chemical ferment acting on the blood, the nerve-centres, and the organs of circulation.

Another reason why cholera-virus and the infective agent, that is, the micro-organism, are two things, and why cholera cannot be an infectious disease of the same group as anthrax, tuberculosis, and others, is that in cholera the body of the patient, notably the choleraic evacuations, do not contain organisms that have any causal relation to the disease. In the true infectious diseases, the specific organism multiplies within the infected borly, is present in the morbid products in great numbers, so that each particle of these products possesses infective property ; but in cholera this is absolutely not the case.

2. This brings us to the second passage referred to in the president's address, namely: The intestinal discharges are the means by which the disease is conveyed from the sick to the healthy. What is the evidence on which an assertion like this one is based? It is well known that linen soiled with choleraic evacuations has been capable of conveying infection; there are, likewise, several well established cases where water, into which choleraic fæcal matter had found entrance, and which been drank, had produced infection. But does this prove that the choleraic evacuations contain the virus, or even the infective agency? By no means; for is it not known that linen and articles that had not been soiled by choleraic evacuations have also been able to convey infection? Is there not available an overwhelming amount of evidence, to show that water that had become contaminated with choleraic dejecta, either directly by the evacuations, or indirectly by the washing of linen soiled with choleraic evacuations, when drunk by a large number of persons, did not produce infection. There is hardly a tank, a ditch, or pool, in town or village in India, at any rate in Bengal, but where, at ordinary non-epidemic times, isolated cases of cholera occur where the choleraic evacuations of these cases find access into the water; and although this is drank, or used for all kinds of domestic purposes by a large number of people living around these tanks, still no infection occurs. If the choleraic evacuations contained the virus, there ought to be-owing to the habits of the natives in India-severe epidemics of cholera, continually, in almost every town of Bengal, and outside. It follows, then, from this, that in those instances where linen soiled with choleraic evacuations, or water contaminated with choleraic evacuations, had conveyed infection, it was not the choleraic evacuations perse, but something added to it, which produced infection. And this something cannot be contained in the evacuations per se, but is something extraneous, seeing that in an overwhelming number of instances the evacuations themselves prove harmless. It further follows from this, that the view of the "contagionists," according to whom cholera is directly communicable from the sick to the healthy by means of the evacuations, cannot by any means be correct. How is it possible to reconcile this view of the contagionists with the well known facts that, when in any cantonment in India cholera appears amongst the troops, they are moved into camp, and although those that have been infected in the cantonment carry the disease into camp, still no new cases occur; how is one to reconcile this view of the contagionists with the well known fact that the attendants on cholera-patients, all those that handle the evacuations, the patients of the wards amongst whom cholera-cases are distributed, remain unaffected ? Cases of cholera amongst hospital-attendants, both those that attend cholerapatients, as well as those that do not, may, and do, occur in epidemics; but then the hospital itself has, like any other house, become a cholera-locality (Pettenkofer).

The fact as to immunity of certain localities, Versailles, Lyons, Birmingham, and others, speaks the same language. Isolated choleracases imported into these localities remain isolated, although the cholera-evacuations are there, and are not disposed of in any manner different from other localities in which such cases lead to an epidemic.

It follows from all this, that the infective agent and virus are not present, and are not necessarily dependent on the choleraic evacuations, but that they are present in a soil extraneous to the body of a patient, and dependent on locality, and, of course, on season.

The contagionists make the perfectly gratuitous assumption that cholera is a directly communicable disease. Of course, if the choleraic infective agent be a bacterium present in the cholera-evacuations, then it follows as a necessary corollary that the disease must be directly

contagious. But this seems to be putting the cart before the horse The evidence that cholera is not and cannot be a directly communicable disease is overwhelming, and therefore any bacterium, however peculiar, present in the choleraic evacuations cannot be connected with the infective agent.

What are the facts brought forward by the contagionists, such as Koch?

The comma-bacillus of Koch is a curved rod of almost uniform thickness, sometimes slightly pointed at the extremities, its length about half that of a tubercle-bacillus, its thickness about the same as that of

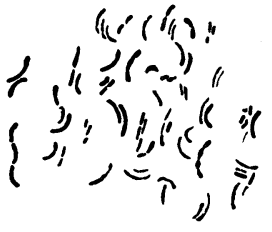

Fig. 1.

From a preparation of mucus-flakes of the fluid in the ileum of a case of typical cholera. Magnifying power about $i 00$. Numbers of comma-bacilli of different lengths are shown amongst numbers of small straight bacilli.

the latter. But the comma-bacilli vary in curvature and length within considerable limits, some being just curved while others are almost semicircular, some being iwice and three times as long as

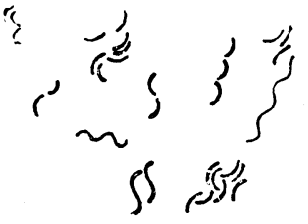

Fici, ?

From an artificial cultivation of chole. raic comma-bacilli in gelatine pepraic comma-bacilli in gelatine peptone. Magnifying power 700 . Most of these are single curved bacteria, a few are joined end to end in twos, thus forming S-shaped organisms; and $a$ few are in chains of several placed end to end. mixture, kept at ordinary temperature, length-divisions may be observed, see Fig. 3. After transverse division they may remain joined end to end, and then forming an S-shaped corpuscle. But sometimes, particularly in artificial cultivations in broth and in liquefied gelatine after several days, they remain joined end to end even after repeated division, and then form either a wavy or spirallike organism. But the type is represented by a single curved rod. For this reason it is not correct to speak of them as comma-bacilli nor as spirilla, since they correspond to what is generally considered a vibrio.

The comma-bacilli are present, amongst many other putrefactive bacteria, in very varying numbers in the choleraic evacuations, sometimes very scarce, sometimes numerous; in the mucus-flakes taken from the lower part of the ileum of some typical rapidly fatal cases of cholera very soon after death they were present in small numbers; in the unper part of the ileum and in the jejunum they are either very scarce or altogether absent. The longer the examination is delayed, of course within certain limits, the more probably are the commabacilli found abundantly in the flakes, but not to the exclusion of other bacteria. They are totally absent from the mucous membrane itself, inclusive of the epithelium of the surface, loosened but not detached. No organisms of any kind are found in the tissue of the intestine, in the blood, and other tissues; putrefactive bacteria, including commabacilli, are capable of growing after death into the clefts and spaces of the intestinal wall from the internal cavity. ${ }^{1}$ (English Cholera Commission.)

In this respect, it must be mentioned that, when they are thus present in the tissue of the ileum, there is no difficulty of dernonstrating them in sections stained with the ordinary aniline dyes. But there came a number of acute typical cases of cholera under observation, where sections of the lower part of the ileum tained with a great variety of aniline dyes in various modifications (Ehrlich's Weigert's, Koch's, and others), in single and double staining, did not reveal a single comma-bacillus or any other bacterium in the tissue of the ileum, not even in the superficial epithelium, which had become loosened, but not yet quite detached. 
The mucus-flakes of the small intestine, taken from a typical rapidly fatal case immediately after death, contain, besides detached epithelial cells, numbers of lymph-corpuscles, some perfect, others swollen up and disintegrating. Soon after death all disintegrate. These lymph-corpuscles or mucus-corpuscles contain, in varying numbers within their protoplasm, straight minute bacilli much smaller

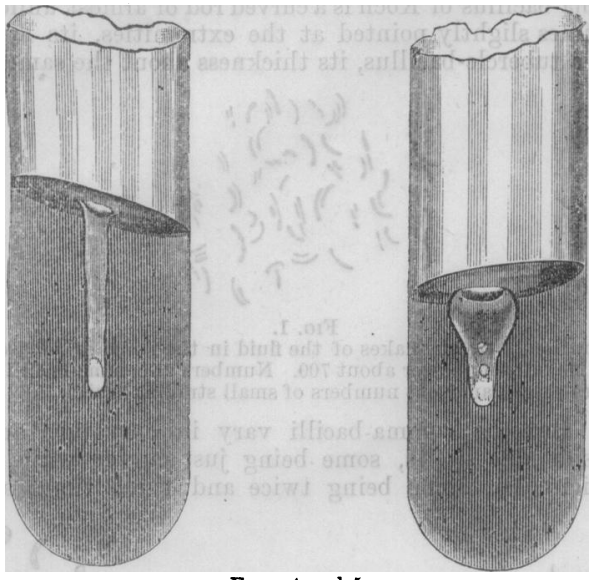

Fias. 4 and 5 .

Pare cultivation of choleraic comma-bacilli in gelatine-peptone-broth. The two tubes had been inoculated at the same time with the same comma-bacilli, and were kept under precisely the same conditions. In both, the surface of growth is marked by a depression. At the bottom of the growth is a whitish precipitate of masses of comma-bacilli. The rest of the channel is filled with almost clear liquefied gelatine.

than the comma-bacilli, being one-half or a fourth their length, and more or less pointed. These small straight bacilli are non-motile, they are never missed in a free state in the mucus-flakes, and when grown artificially they form spores. Neither the comma-bacilli nor these small bacilli show in their mode of growth, in artificial cultivations in various media, greater peculiarities than other putrefactive bacteria. The peculiar mode of growth of the comma-bacilli in gelatine mixtures and in Agar-Agar mixtures is shown in the accompanying woodcuts. ${ }^{2}$

Both the comma-bacilli and small straight bacilli grow well in alkaline and neutral media, and are not killed by acids, although they do

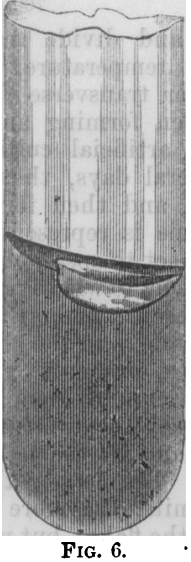

Pure cultivation of choleraio comma-bacilli in gelatine-peptone-broth. The inoculation had been made, not in a channel, as in the previous figures, but on the surface. There is also here a depression of the growth on the surface, and in the extent of the growth the gelatine is liquefied, with a whitish precipitate at the bottom.

not show growth in them, or only to a very limited degree. ${ }^{3}$ Neither the comma-bacilli nor the straight small bacilli can be considered as connected with the cause of cholera.

2 Mr. Watson Cheyne, in a subsequent criticism, maintained that these short straight bacilli are only young forms of comma-bacilli ; this I consider a perfectly gratuitous statement. I am quite sure that, in artificial cultivations of pure comma-bacilli, there is nothing of the sort; when straight bacilli occur, they are due to contamination.

3 This assertion is based on the following experiments. 1. Choleraic commahacilli have been grown in meat-broth peptone-solutions of distinctly acid reaction The growth was limited, but there was growth. 2. Choleraic comma-bacilli were The growth was limited, but there was groteen minutes. With a droplet of this
The results of the experiments performed by Nicat and Rietsch, by Koch and others, namely, death following in some of the animals after injection of choleraic mucus-flakes, or of cultivations of conmabacilli, into the cavity of the small intestine, were not due to cholera, but were clearly due either to the operation, or to septicæmic poison. ing. ${ }^{4}$ Rodents, carnivorous animals, and monkeys, must be considered unsusceptible to cholera.

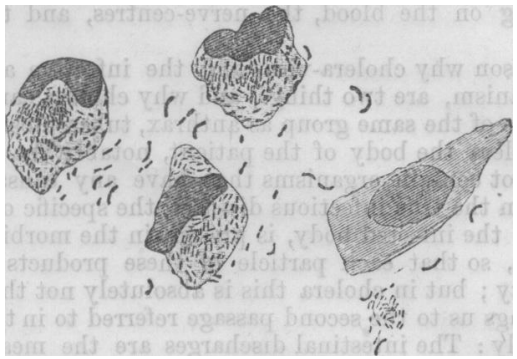

Fig. 7 .

From a preparation of mucus-flakes of the ileum of a case of typical cholera Comma-bacilli and miunte straight bacilli, singly or in masses. Three lymphcorpuscles containing in their interior numerous small straight bacilli.

Comma-bacilli of various species have been discovered in other diseases of the alimentary canal, in the fluid of the mouth of normal persons (Lewis), in old cheese (Deneke). The comma-bacilli found by Finkler and Prior in cholera nostras, differ in mode of growth from Koch's comma-bacilli of cholera; so do those found in diarrhœa due to other causes; but those of the fluid of the mouth are identical with Koch's comma-bacilli in many respects.

mixture, culture-tubes containing alkaline meat-broth, or alkaline meat-broth peptone, were then inoculated, and exposing these to a temperature of $35^{\circ}$ to $38^{\circ} \mathrm{C}$. for two to three days, a copious growth of typical comma-bacilli will be found. From these tubes, ind a mude of nutritive gelatine, and pure typical growths of comma-bacilli are the result But inoculating with the comma bacili grow baying the that the the Saying, then, that the choleraic comma-bacilli do not grow in faintly acid metlia (Koch) is altogether a different thing from saying that the choleraic comma-bacill re killed by weak acids. From the fact that the comma-bacilli of the saliva cannot be made to grow in alkaline nutritive gelatine, no conclusion can be drawn as to their being different in mode of growth from the choleraic conma-bacilli The above observations on the choleraic comma-bacilli in $\mathrm{HCl}$. clearly establishec this ; but I have succeeded in producing growths of the salivary comma-bacill in alkaline nutritive gelatine, which growth presents all the characters typical of the choleraic comma-bacilli. Such growth was accomplished by growing the salivary comma-bacilli first in neutral medium for one or two generations, and then from this, inoculation of alkaline gelatine was established.

4 The experiments inade in India, injection of choleraic mucus-flakes (full of comma-bacilli), recent and old, and of pure cultivations of comma-bacilli, into the duodenum, blood, peritoneal cavity, etc., of rabbits, cats, ldogs, and monkeys, did not produce any result. Likewise Professor Horsley made, at the Brown Institution, London, a series of such experiments (injection of pure cultivations of choleraic comma-bacilli, recent and old, into the duodenum of rodents, and of a large number of dogs, the operation being performed antiseptically), and no result was nuce of was produced. Mr. Watson Cheyne subsequently stated that, in a large number of experiments performed by him on rabbits, he had two positive cases-two nimals did die with diarrhœa. It would be most important to know whether $\mathrm{Mr}$ Cheyne made these experiments antiseptically, and what was the duration of the illness. That rodents, particularly rabbits, occasionally die in consequence of light operations, and that such animals show in 50 per cent. of the cases diarrhoea, is a familiar fact to all who have had experience in these matters, and likewise that such animals do not in many instances contain in the blood or tissues any trace of bacteria. But granting, for the sake of argument, that the animals operated upon died in consequence of the injection with the comma-bacilli, does this prove that the comma-bacilli are the cause of cholera in man? Not at all. I refer to the observations of Bienstock, who isolated from normal human fwces a bacillus which proved fatal to some rodents.

Accessory Mammary Glands. - Luschka has classified supernumerary mammary glands under two distinct divisions: mamma erratice and mamma aberrans. Mamma erratica is a true supernumerary mammary gland, developed on any part of the body excepting its normal position, the front of the thorax. Mamma aberrans signifies a gland that lies on or near the pectoralis major, but has a distinct outlet for its ducts, separate from the true nipple. This condition is rare. Dr. Cohn exhibited a case before the Berliner Medizinische Gesellschaft, on February 25th. It was not an instance of milk-fistula, which generally arises from a wound, or from abscess. The patient, in this case, it is true, had inflammation of the natural gland on the left side, and quite accidentally found out that milk dribbled away from the axilla. On careful examination, a duct was found opening in the axilla. It was covered with skin, and, on pressure of the integument around it, milk squirted out. 